\title{
Treatment of community-onset pneumonia in neutropenic cancer patients: $\beta$-lactam monotherapy versus combination antibiotic regimens
}

Hyeri Seok ${ }^{1,2+}$, Jae-Hoon $\mathrm{Ko}^{2+}$, Kyong Ran Peck ${ }^{2 *}$ (D) Ji-Yeon $\mathrm{Kim}^{2}$, Ji Hye Lee ${ }^{2}$, Ga Eun Park², Sun Young Cho ${ }^{2}$, Cheol-In Kang ${ }^{2}$, Nam Yong Lee ${ }^{3}$ and Doo Ryeon Chung ${ }^{2}$

\begin{abstract}
Background: Although $\beta$-lactam monotherapy may be sufficient in non-critically ill patients with communityacquired pneumonia, the value of combination antibiotic regimens in community-onset neutropenic pneumonia remains unclear.

Methods: A retrospective cohort study was conducted to compare the effects of combination antibiotic regimens to those of $\beta$-lactam monotherapy in cancer patients with community-onset neutropenic pneumonia. Electronic medical records of patients diagnosed with community-onset neutropenic pneumonia between March 1995 and February 2015 at a tertiary care center were reviewed.

Results: During the study period, 165 cancer patients with community-onset neutropenic pneumonia were identified. Seventy-two patients received $\beta$-lactam monotherapy and 93 received combination therapy ( $\beta$-lactam plus either a macrolide or fluoroquinolone). Causative pathogens were identified in $27.9 \%$ of the patients, and only two were positive for atypical pathogens. Although 30-day mortality was higher in the $\beta$-lactam group (15.3\% versus 4.3\%; $P=0.015)$, combination therapy was not associated with a statistically significant survival benefit in the multivariate analysis (hazard ratio $0.85,95 \%$ confidence interval $0.20-3.67 ; P=0.827$ ). Duration of neutropenia, $C$-reactive protein level, and Multinational Association for Supportive Care in Cancer risk index were significant factors for 30-day mortality. In a subgroup analysis of patients treated with cefepime, the most frequently used $\beta$-lactam (63.0\%), combination therapy also showed no significant survival benefit.
\end{abstract}

Conclusions: Combination antibiotic regimens were not associated with a survival benefit over $\beta$-lactam monotherapy in the treatment of community-onset neutropenic pneumonia. Unnecessary combination therapy should be reconsidered in cancer patients who are at high risk for adverse drug reactions and colonization with multidrug resistant organisms.

Keywords: Febrile neutropenia, Pneumonia, Cancer, Combination antibiotic regimens

\footnotetext{
* Correspondence: krpeck@skku.edu

${ }^{\dagger}$ Hyeri Seok and Jae-Hoon Ko contributed equally to this work.

2Division of Infectious Diseases, Department of Medicine, Samsung Medical

Center, Sungkyunkwan University School of Medicine, 81 Irwon-ro,

Gangnam-gu, Seoul 06351, Republic of Korea

Full list of author information is available at the end of the article
}

(c) The Author(s). 2019 Open Access This article is distributed under the terms of the Creative Commons Attribution 4.0 International License (http://creativecommons.org/licenses/by/4.0/), which permits unrestricted use, distribution, and reproduction in any medium, provided you give appropriate credit to the original author(s) and the source, provide a link to the Creative Commons license, and indicate if changes were made. The Creative Commons Public Domain Dedication waiver (http://creativecommons.org/publicdomain/zero/1.0/) applies to the data made available in this article, unless otherwise stated. 


\section{Background}

Febrile neutropenia, an inevitable side effect of chemotherapy, contributes to high mortality among cancer patients [1]. Among various clinical presentations of febrile neutropenia, pneumonia is considered high-risk [2]. Guidelines do not specify empirical antibiotics for the treatment of neutropenic pneumonia, i.e. communityonset pneumonia with febrile neutropenia. Although the European Society for Medical Oncology guidelines recommend coverage of atypical pathogens by adding macrolide antibiotics in managing neutropenic pneumonia, the level of evidence and grade of recommendation are very low [3]. Most clinicians choose a combination of an antipseudomonal $\beta$-lactam with azithromycin or fluoroquinolone targeting neutropenic fever and community-acquired pneumonia (CAP). However, the indiscriminate use of macrolide or fluoroquinolone in cancer patients receiving chemotherapy may result in unexpected side effects such as cardiovascular events and acquisition of resistance [47]. This study aimed to answer the question of whether combination therapy is necessary in patients with neutropenic pneumonia.

In the case of CAP, there has been already controversy about whether to start combination therapy as empirical antibiotics. The addition of macrolide or fluoroquinolone is based on the treatment for an atypical pathogen, antibiotic synergism, and anti-inflammatory effect. However, several studies have shown that $\beta$-lactam monotherapy is non-inferior to combination therapy [8-10]. Some have suggested that monotherapy may be considered in patients with low-risk CAP $[11,12]$. Likewise, questions may arise as to whether combination therapy should be considered when neutropenic pneumonia is diagnosed in cancer patients who are receiving chemotherapy but with good performance status. To further investigate this question, we compared the effects of combination antibiotic regimens to those of $\beta$-lactam monotherapy in cancer patients with community-onset neutropenic pneumonia who were managed at a tertiary care center during a 20-year period.

\section{Methods}

\section{Study design and population}

A retrospective cohort study was conducted to compare the effects of combination antibiotic regimens to those of $\beta$-lactam monotherapy for the treatment of communityonset pneumonia in neutropenic cancer patients. We reviewed the electronic medical records of individuals who were admitted and diagnosed with pneumonia between March 1995 and February 2015 at Samsung Medical Center, a 1950-bed tertiary care university hospital in Seoul, Republic of Korea. Adult (age $\geq 18$ years) cancer patients who were diagnosed with community-onset neutropenic pneumonia during the study period were included in the analysis. We exclusively included chemotherapyinduced neutropenia in the present study, and underlying cancers included solid cancers, lymphoma, and multiple myeloma. Hematologic malignancies such as acute leukemia, aplastic anemia, or myelodysplastic syndrome that accompany prolonged neutropenia due to intensive chemotherapy or the nature of the disease, were excluded from the analysis because prolonged neutropenia, a strong prognostic factor of febrile neutropenia, may obscure the effect of antibiotics [1, 13, 14]. Patients with lymphoma and multiple myeloma who underwent bone marrow transplantation were also excluded for the same reason. The study population was divided into two groups according to antibiotic regimen. Patients who were re-admitted 7 days after discharge were excluded because they were assumed to have hospital-acquired pneumonia. The combination group included patients who were treated with a combination of a $\beta$-lactam and a macrolide or fluoroquinolone, and the $\beta$-lactam group included patients who received $\beta$-lactam therapy only. Additional use of other classes of antibiotics that do not have activity for atypical bacterial pathogens, such as aminoglycosides or vancomycin, was not considered when classifying study groups. The primary outcome was 30-day all-cause mortality, and secondary outcomes were 90-day all-cause mortality, length of hospital stay, and complications. The study protocol was approved by the Institutional Review Board of Samsung Medical Center.

\section{Data collection}

Retrospectively collected data included demographics, underlying diseases, clinical presentations and outcomes, laboratory test results, types and duration of antibiotic therapy, and microbiologic data. Microbiologic data included cultures of blood and respiratory specimens, pneumococcal and Legionella urinary antigen, influenza antigen, and mycoplasma antibody. The severity of neutropenic pneumonia was estimated using the CURB-65 score and the Multinational Association for Supportive Care in Cancer (MASCC) risk index $[15,16]$.

\section{Definitions}

Pneumonia was defined as the presence of parenchymal infiltration on chest radiography (newly appeared infiltration or aggravation of preexisting lesions) with relevant respiratory symptoms, including cough, sputum production, shortness of breath, or pleuritic chest pain [17]. Pneumonia was considered to be of communityonset if it developed in the community or within $48 \mathrm{~h}$ after hospital admission $[17,18]$. Neutropenia was defined as an absolute neutrophil count $<500$ cells $/ \mathrm{mm}^{3}$ or an absolute neutrophil count expected to decrease to $<500$ cells $/ \mathrm{mm}^{3}$ during the next $48 \mathrm{~h}$ [2]. Fever was defined as body temperature $\geq 38.0^{\circ} \mathrm{C}$. The response to 
chemotherapy was evaluated according to the World Health Organization criteria or Response Evaluation Criteria in Solid Tumors guidelines [19-21]. The mycoplasma antibody test was considered positive if a single titer was highly elevated $(\geq 1: 320)$ or a four-fold increase in the titer was detected in a follow-up sample [22].

\section{Statistical analyses}

The sample size calculation for the study resulted in a sample size of 134 patients who were to be in a 1:1 allocation ratio to achieve a power of $95 \%$. To compare the two groups, the Pearson $x^{2}$ test and Fisher's exact test were used for categorical variables, and Student's $t$-test and the Mann-Whitney $U$ test for continuous variables. The Cox proportional hazard model was used to examine the associations of the antibiotic regimens with 30and 90-day mortality in neutropenic pneumonia after adjusting for potential confounding factors. All collected variables with any relevance to outcomes were evaluated by univariate analysis, and those with statistical significance were included in the multivariate analysis. All $P$ values were two-tailed, and those $<0.05$ were considered to be statistically significant. All statistical analyses were performed using SPSS Statistics version 20.0 for Windows (IBM Corp., Armonk, NY, USA).

\section{Results}

Baseline characteristics, antibiotic types, severity, and cancer status of neutropenic pneumonia

During the study period, 165 cancer patients with community-onset neutropenic pneumonia were identified: 72 were classified in the $\beta$-lactam group and 93 in the combination group. Blood cultures were performed in $100 \%$ of the enrolled patients and cultures of lower respiratory specimens in $99.0 \%$ of the patients. Causative pathogens of neutropenic pneumonia were identified in $27.9 \%$ of the patients, of which Streptococcus pneumoniae was the most common followed by Pseudomonas aeruginosa, Staphylococcus aureus, and Klebsiella pneumoniae (23.9, 19.6, 15.2, and 13.0\%, respectively; Additional file 1: Table S1). Serologic tests for Mycoplasma pneumoniae were conducted in 20 patients (12.1\%), two of which yielded positive results. The urinary antigen tests for legionella were performed in 75 patients (45.5\%), and all showed results. No patient received oral prophylactic antibiotics before the onset of neutropenic fever.

The baseline characteristics, types of antibiotics, and the severity of neutropenic pneumonia are presented in Table 1. Baseline characteristics were similar between the two groups, except for male predominance in the $\beta$ lactam group and a higher prevalence of chronic renal disease in the combination group. Ceftazidime was used more frequently in the $\beta$-lactam group, while piperacillin- tazobactam was used more in the combination group. Ceftazidime was administered with tobramycin for an initial 3-5 days, and $23.6 \%$ of the $\beta$-lactam group and $19.4 \%$ of the combination group used an initial vancomycin combination $(P=0.507)$. In the combination group, azithromycin was most frequently used for coverage of atypical pathogens, followed by levofloxacin, ciprofloxacin, and moxifloxacin $(75.3,11.8,8.6$, and $4.3 \%$, respectively). The duration of antibiotic administration was similar between the two groups. Severity variables, including the presence of bacteremia, duration of neutropenia, Creactive protein (CRP) level, CURB-65 score, and MASCC risk index were not statistically different between the two groups (all $P>0.05$ ).

Types and status of cancers in patients with neutropenic pneumonia were not differently distributed between the two groups (Table 2 , all $P>0.05$ ). Lung cancer was the most common type of cancer, followed by lymphoma and breast cancer $(44.8,14.6$, and $11.5 \%$, respectively). Sixty-seven patients (40.6\%) received chemotherapy for curative purposes, while the remainders (59.4\%) were in palliative settings. Among patients in palliative settings, more than half $(54.1 \%)$ experienced progression or relapse of underlying cancers despite previous chemotherapy.

\section{Outcomes and complications of patients with neutropenic pneumonia}

The outcomes and complications of patients with neutropenic pneumonia are summarized in Table 3 . In the $\beta$-lactam group, 30 -day $(15.3 \%$ versus $4.3 \%, P=0.015)$ and 90 -day all-cause mortality $(20.8 \%$ versus $7.5 \%, P=$ 0.013 ) were significantly higher. Length of hospital stay, pneumonia recurrence, and complications and adverse effects, including complicated pleural effusion, Clostridium difficile-associated colitis, and cardiac events, were similar between the two groups (all $P>0.05$ ).

To identify potential confounding factors of 30-day all-cause mortality, all relevant variables were evaluated in a univariate fashion using the Cox proportional hazard model (Table 4). Underlying lung cancer, palliative setting, neutropenia duration, CRP level, MASCC risk index, combination therapy, and the usage of cefepime and carbapenem were statistically significant factors of 30-day all-cause mortality in the univariate analysis. In the multivariate analysis of these variables, combination therapy did not maintain a significant association (hazard ratio [HR] $0.85,95 \%$ confidence interval [CI] 0.20-3.67; $P$ $=0.827$ ). Duration of neutropenia (HR 1.27, 95\% CI 1.051.53; $P=0.013$ ), CRP level (HR 1.06, 95\% CI 1.00-1.11; $P$ $=0.021$ ), and MASCC risk index (HR 0.80, 95\% CI 0.65$0.99 ; P=0.042)$ were persistent statistically significant factors of 30-day all-cause mortality. A multivariate analysis for 90-day all-cause mortality was performed 
Table 1 Baseline characteristics, types of $\beta$-lactams, and severity of patients with neutropenic pneumonia

\begin{tabular}{|c|c|c|c|}
\hline Variables & $\beta$-lactam group $(n=72)$ & Combination group $(n=93)$ & $P$-value \\
\hline \multicolumn{4}{|l|}{ Demographic data } \\
\hline Age, years & $61 \pm 11$ & $62 \pm 10$ & 0.296 \\
\hline Male sex & $59(81.9 \%)$ & $59(63.4 \%)$ & 0.009 \\
\hline Smoking history & $29(40.3 \%)$ & $27(29.0 \%)$ & 0.130 \\
\hline \multicolumn{4}{|l|}{ Comorbid conditions } \\
\hline Hypertension & $14(19.4 \%)$ & $28(30.1 \%)$ & 0.119 \\
\hline Diabetes mellitus & $5(6.9 \%)$ & $12(12.9 \%)$ & 0.212 \\
\hline Cardiovascular diseases & $5(6.9 \%)$ & $2(2.2 \%)$ & 0.130 \\
\hline Chronic lung diseases & $3(4.2 \%)$ & $6(6.5 \%)$ & 0.733 \\
\hline Chronic liver diseases & $3(4.2 \%)$ & $3(3.2 \%)$ & 1.000 \\
\hline Chronic renal diseases & $0(0.0 \%)$ & $6(6.5 \%)$ & 0.036 \\
\hline \multicolumn{4}{|l|}{ Types of $\beta$-lactams } \\
\hline Cefepime & $40(55.6 \%)$ & $64(68.8 \%)$ & 0.080 \\
\hline Ceftazidime $^{a}$ & $20(27.8 \%)$ & $2(2.2 \%)$ & $<0.001$ \\
\hline Piperacillin/tazobactam & $6(8.3 \%)$ & $21(22.6 \%)$ & 0.014 \\
\hline Carbapenem & $6(8.3 \%)$ & $6(6.5 \%)$ & 0.644 \\
\hline Duration of antibiotic administration, days & $12.4(8.9-16.2)$ & $11.7(9.1-14.4)$ & 0.646 \\
\hline \multicolumn{4}{|l|}{ Severity variables } \\
\hline Presence of bacteremia & $16(22.0 \%)$ & $12(12.9 \%)$ & 0.114 \\
\hline Duration of neutropenia, days & $1.5(0.9-3.1)$ & $1.4(1.0-2.9)$ & 0.773 \\
\hline $\mathrm{CRP}, \mathrm{mg} / \mathrm{dL}$ & $15.9(6.5-24.0)$ & $17.0(6.4-25.9)$ & 0.669 \\
\hline CURB-65 & $1(0-2)$ & $1(0-2)$ & 0.898 \\
\hline MASCC risk index & $16(13-18)$ & $16(16-21)$ & 0.059 \\
\hline
\end{tabular}

Data are expressed as number (\%) of patients, mean $\pm \mathrm{SD}$, or median (IQR)

${ }^{a}$ Ceftazidime was administered with tobramycin for an initial 3-5 days

Abbreviations: CRP C-reactive protein, MASCC Multinational Association for Supportive Care in Cancer, SD Standard deviation, IQR interquartile range

(Additional file 2: Table S2). Combination therapy was not significantly associated with 90-day all-cause mortality (HR 0.67, 95\% CI $0.23-2.76 ; P=0.449$ ), while palliative setting of chemotherapy (HR 12.81, 95\% CI 1.78-92.20; $P$ $=0.011)$, duration of neutropenia (HR 1.16, 95\% CI 1.07$1.25 ; P=<0.001$ ), CRP level (HR 1.05, 95\% CI 1.01-1.09; $P=0.020$ ), and MASCC index (HR 0.84, 95\% CI $0.72-$ $0.98 ; P=0.024)$ were statistically related to 90 -day allcause mortality.

\section{Subgroup analysis of cefepime-treated patients with neutropenic pneumonia}

Although we performed a multivariate analysis to adjust for potential confounding factors, the types of $\beta$-lactams were significantly unbalanced between the two groups. To verify the effect of combination therapy on all-cause mortality due to neutropenic pneumonia, we also performed a subgroup analysis controlling for $\beta$-lactam antibiotics, specifically cefepime which made up the highest proportion among the various $\beta$-lactams (63.0\%). The same variables used in the analysis of the original cohort were included in the multivariate analyses for 30- and 90-day all-cause mortality. In the multivariate analyses of the cefepime-treated subgroup, combination therapy was not significantly associated with either 30-day (HR 0.76, 95\% CI 0.06-9.18; $P=0.965$ ) or 90-day all-cause mortality (HR 0.78 , 95\% CI $0.18-$ $3.45 ; P=0.741)$.

\section{Discussion}

This study showed that neither monotherapy nor combination therapy was associated with 30- and 90-day mortality in the treatment of neutropenic pneumonia. Although 30-day and 90-day all-cause mortality were higher in the $\beta$-lactam group than in the combination group before adjustment, this finding suggests that more patients in the $\beta$-lactam group had confounding factors for poor prognosis, including lung cancer, longer duration of neutropenia, or bacteremia, than patients in the combination group.

To our knowledge, this is the first study to compare the clinical efficacy of monotherapy and combination therapy in the treatment of neutropenic pneumonia, extending the concept of CAP. It has been already argued that 
Table 2 Types and status of cancers in patients with neutropenic pneumonia

\begin{tabular}{|c|c|c|c|}
\hline Variables & $\beta$-lactam group $(n=72)$ & Combination group $(n=93)$ & $P$-value \\
\hline \multicolumn{4}{|l|}{ Type of cancer } \\
\hline Head and neck & $3(4.2 \%)$ & $1(1.1 \%)$ & 0.319 \\
\hline Lung & $36(50.0 \%)$ & 38 (40.9\%) & 0.242 \\
\hline Breast & $7(9.7 \%)$ & $12(12.9 \%)$ & 0.526 \\
\hline Esophageal and gastric & $8(11.1 \%)$ & $6(6.5 \%)$ & 0.287 \\
\hline Colorectal & $1(1.4 \%)$ & $5(5.4 \%)$ & 0.233 \\
\hline Hepatobiliary and pancreatic & $0(0.0 \%)$ & $3(3.2 \%)$ & 0.258 \\
\hline Bladder & $1(1.4 \%)$ & $5(5.4 \%)$ & 0.233 \\
\hline Prostate & $0(0.0 \%)$ & $4(4.3 \%)$ & 0.133 \\
\hline Melanoma and sarcoma & $1(1.4 \%)$ & $5(5.4 \%)$ & 0.233 \\
\hline Lymphoma and myeloma & $15(20.8 \%)$ & $13(14.0 \%)$ & 0.245 \\
\hline \multicolumn{4}{|l|}{ Cancer status } \\
\hline Curative setting & $32(44.4 \%)$ & $35(37.6 \%)$ & 0.377 \\
\hline Neoadjuvant and adjuvant & $12(16.7 \%)$ & $20(21.5 \%)$ & 0.436 \\
\hline Definitive & $20(27.8 \%)$ & $15(16.1 \%)$ & 0.069 \\
\hline Palliative setting ${ }^{a}$ & $40(55.6 \%)$ & $58(62.4 \%)$ & 0.377 \\
\hline Complete response & $1(2.5 \%)$ & $0(0.0 \%)$ & 0.436 \\
\hline Partial response & $2(5.0 \%)$ & $4(6.9 \%)$ & 0.697 \\
\hline Stable disease & $0(0.0 \%)$ & $4(6.9 \%)$ & 0.133 \\
\hline Progressed or relapse & $22(55 \%)$ & 31 (53.4\%) & 0.705 \\
\hline Before evaluation & $15(37.5 \%)$ & $19(32.8 \%)$ & 0.949 \\
\hline
\end{tabular}

Data are expressed as number (\%) of patients

${ }^{a}$ The response to chemotherapy was evaluated according to the World Health Organization (WHO) criteria or Response Evaluation Criteria in Solid Tumors (RECIST) guidelines

monotherapy is sufficient for mild cases of CAP without neutropenia $[11,12]$. The reasons for combination treatment is to cover atypical pathogens, antibiotics synergism, or anti-inflammatory effects. However, in fact, the proportion of atypical pathogens is known to be low in non-critically ill patients with CAP $[8,23]$. A lack of synergistic effect between macrolide and $\beta$-lactam against $S$. pneumoniae has been described [24-26]. To date, no studies have shown the direct association between combination therapy and anti-inflammatory effect. Several studies have proven that $\beta$-lactam monotherapy is noninferior to combination treatment $[8-10,26]$.

The concept that combination treatment may be unnecessary can be applied to neutropenic pneumonia.

Table 3 Outcomes and complications of patients with neutropenic pneumonia

\begin{tabular}{llll}
\hline Variables & $\beta$-lactam group $(n=72)$ & Combination group $(n=93)$ & $P$-value \\
\hline 30-day all-cause mortality & $11(15.3 \%)$ & $4(4.3 \%)$ & 0.015 \\
90-day all-cause mortality & $15(20.8 \%)$ & $7(7.5 \%)$ & 0.013 \\
Length of hospital stay, days $_{\text {Recurrence of pneumonia }}{ }^{a}$ & $5.7(4.4-10.9)$ & $6.0(4.3-8.6)$ & 0.632 \\
Complications and adverse effects $^{\mathrm{a}}$ & $4(5.6 \%)$ & $3(3.2 \%)$ & 0.700 \\
$\quad$ & & & $2(2.2 \%)$ \\
$\quad$ Complicated pleural effusion & $1(1.4 \%)$ & $0(0.0 \%)$ & 0.081 \\
$\quad$ CDAD & $3(4.2 \%)$ & $4(4.3 \%)$ & 0.730 \\
$\quad$ Others $^{c}$ & $4(5.6 \%)$ & $7(7.5 \%)$ & 0.394 \\
\hline
\end{tabular}

Data are expressed as number (\%) of patients or median (IQR)

aithin 90 days

${ }^{\mathrm{b} C a r d i a c}$ events included arrhythmia, heart failure, and myocardial infarction

'Others included diarrhea, gastrointestinal bleeding, pneumothorax, epilepsy, and back pain

Abbreviations: CDAD Clostridium difficile-associated diarrhea, IQR interquartile range 
Table 4 Multivariate analysis of 30-day all-cause mortality due to neutropenic pneumonia

\begin{tabular}{|c|c|c|c|c|}
\hline \multirow[t]{2}{*}{ Variables } & \multicolumn{2}{|l|}{ Univariate analysis } & \multicolumn{2}{|l|}{ Multivariate analysis } \\
\hline & $\mathrm{HR}(95 \% \mathrm{Cl})$ & $P$-value & $\mathrm{HR}(95 \% \mathrm{Cl})$ & $P$-value \\
\hline Lung cancer & $3.55(1.13-11.15)$ & 0.030 & $1.60(0.30-8.63)$ & 0.586 \\
\hline Palliative setting & $4.83(1.09-21.43)$ & 0.038 & $58.61(0.26-13,356.90)$ & 0.142 \\
\hline Duration of neutropenia & $1.08(1.03-1.15)$ & 0.003 & $1.27(1.05-1.53)$ & 0.013 \\
\hline CRP & $1.04(1.00-1.08)$ & 0.035 & $1.06(1.00-1.11)$ & 0.021 \\
\hline MASCC risk index & $0.80(0.68-0.95)$ & 0.010 & $0.80(0.65-0.99)$ & 0.042 \\
\hline Combination therapy & $0.27(0.09-0.85)$ & 0.025 & $0.85(0.20-3.67)$ & 0.827 \\
\hline \multicolumn{5}{|l|}{ Types of $\beta$-lactams } \\
\hline Cefepime & $0.28(0.10-0.81)$ & 0.019 & $0.64(0.17-2.46)$ & 0.518 \\
\hline Ceftazidime & $2.05(0.58-7.25)$ & 0.268 & & \\
\hline Piperacillin-tazobactam & $1.42(0.40-5.02)$ & 0.589 & & \\
\hline Carbapenem & $5.95(1.89-18.70)$ & 0.002 & $0.28(0.01-50.00)$ & 0.631 \\
\hline
\end{tabular}

Abbreviations: Cl confidence interval, CRP C-reactive protein, HR hazard ratio, MASCC Multinational Association for Supportive Care in Cancer

Cancer patients are at high risk for adverse drug reactions such as gastrointestinal and cardiogenic toxicity. In addition, cancer patients are more likely to be colonized with multi-drug resistant organisms with exposure to antibiotics. These problems can be overcome without the use of unnecessary antibiotics. This study showed that the rate of atypical pathogens was low although not all patients were examined. $\beta$-lactam monotherapy was not associated with 30- and 90-day mortality in the multivariate analysis. Rather, predictable variables such as duration of neutropenia, CRP level, and MASCC risk index were associated with mortality in neutropenic pneumonia. This result may support the fact that monotherapy is sufficient in non-critically ill patients with neutropenic pneumonia.

Due to the extended study period of 20 years, there were considerable differences in the types of $\beta$-lactams between the two groups. The preferred regimen for neutropenic pneumonia at our center was changed from ceftazidime combined with tobramycin to cefepime after 2002 due to increasing ceftazidime-resistance [27]. Instead of a tobramycin combination, clinicians tended to select either a macrolide or fluoroquinolone combinations to cover atypical pathogens in neutropenic pneumonia. As these changes in preferred regimens depending on the period may have introduced some bias, we performed a subgroup analysis of cefepimetreated patients, and also found no difference in outcomes between the two groups, supporting the results of the main cohort.

There are several limitations to our study. First, the 20 -year study period may lead to heterogeneity in the study population. However, we inevitably expanded the study period to obtain statistical significance in comparing the two groups. We conducted both multivariate analyses to adjust for potential confounding factors and subgroup analysis of cefepime-treated patients to overcome this limitation. Second, as a limitation of retrospective studies, certain criteria have not been applied to the selection of the antibiotics, and the test results for diagnosis of pneumonia. Finally, screening for atypical pathogens was not performed in all patients and the Legionella test in this study covered only serotype 1 . This is also the limitation of retrospective study, and efforts to find causative organisms of pneumonia through the tests including urinary antigen detection, respiratory virus PCR panels, sputum cultures are needed. Additional prospective studies are needed to modify this limitation.

In conclusion, this study revealed no differences in 30and 90 -day mortality between $\beta$-lactam monotherapy and combination therapy in cancer patients with non-severe community-onset neutropenic pneumonia. Monotherapy can reduce the chance of unwanted drug adverse reactions and acquisition of antibiotic resistance. Unnecessary combination therapy should be reconsidered in neutropenic pneumonia. Future studies are needed with better evaluations for atypical pathogens.

\section{Additional files}

Additional file 1: Table S1. Causative pathogens of neutropenic pneumonia. (DOCX $15 \mathrm{~kb}$ )

Additional file 2: Table S2. Multivariate analysis of 90-day all-cause mortality due to neutropenic pneumonia. (DOCX $15 \mathrm{~kb}$ )

Abbreviations

CAP: Community-acquired pneumonia; Cl: Confidence interval; CRP: Creactive protein; HR: Hazard ratio; MASCC: Multinational association for supportive care in cancer

Authors' contributions

All authors have read and approved the manuscript. The authors were solely responsible for the conception and performance of the study and for writing 
this manuscript. Conceptualization: HS, JHK, KRP. Data collection: HS, JYK, JHL, GEP. Data analysis: HS, JHK, KRP, SYC, CIK. Writing original draft: HS, JHK. Writing review \& editing: HS, JHK, KRP, JYK, JHL, GEP, SYC, CIK, NYL, DRC.

\section{Funding}

No funding was provided for this study.

\section{Ethics approval and consent to participate}

Not applicable.

\section{Consent for publication}

Not applicable.

\section{Competing interests}

The authors of this manuscript have conflicts of interest to disclose as described by BMC Infectious Diseases.

\section{Author details}

${ }^{1}$ Division of Infectious Diseases, Department of Medicine, Korea University Ansan Hospital, Ansan, Gyeonggi-do, Republic of Korea. ${ }^{2}$ Division of Infectious Diseases, Department of Medicine, Samsung Medical Center, Sungkyunkwan University School of Medicine, 81 Irwon-ro, Gangnam-gu, Seoul 06351, Republic of Korea. ${ }^{3}$ Department of Laboratory Medicine, Samsung Medical Center, Sungkyunkwan University School of Medicine, Seoul, Republic of Korea.

\section{Received: 26 November 2018 Accepted: 21 May 2019}

Published online: 05 June 2019

\section{References}

1. Kuderer NM, Dale DC, Crawford J, Cosler LE, Lyman GH. Mortality, morbidity, and cost associated with febrile neutropenia in adult cancer patients. Cancer. 2006:106(10):2258-66.

2. Freifeld AG, Bow EJ, Sepkowitz KA, Boeckh MJ, Ito Jl, Mullen CA, et al. Clinical practice guideline for the use of antimicrobial agents in neutropenic patients with cancer: 2010 update by the infectious diseases society of america. Clin Infect Dis. 2011:52(4):e56-93.

3. de Naurois J, Novitzky-Basso I, Gill MJ, Marti FM, Cullen MH, Roila F, et al. Management of febrile neutropenia: ESMO Clinical Practice Guidelines. Ann Oncol. 2010;21(Suppl 5):v252-6.

4. Fuller JD, Low DE. A review of Streptococcus pneumoniae infection treatment failures associated with fluoroquinolone resistance. Clin Infect Dis. 2005;41(1):118-21.

5. Malhotra-Kumar S, Lammens C, Coenen S, Van Herck K, Goossens H. Effect of azithromycin and clarithromycin therapy on pharyngeal carriage of macrolide-resistant streptococci in healthy volunteers: a randomised, double-blind, placebo-controlled study. Lancet. 2007;369(9560):482-90.

6. Ray WA, Murray KT, Hall K, Arbogast PG, Stein CM. Azithromycin and the risk of cardiovascular death. N Engl J Med. 2012;366(20):1881-90.

7. Schembri S, Williamson PA, Short PM, Singanayagam A, Akram A, Taylor J, et al. Cardiovascular events after clarithromycin use in lower respiratory tract infections: analysis of two prospective cohort studies. Bmj. 2013;346:f1235.

8. Postma DF, van Werkhoven CH, van Elden L, Thijsen SF, Hoepelman Al, Kluytmans JA, et al. Antibiotic treatment strategies for community-acquired pneumonia in adults. N Engl J Med. 2015;372(14):1312-23.

9. van der Eerden MM, Boersma WG. Single versus combination antibiotic therapy in adults hospitalised with community-acquired pneumonia. Thorax 2013;68(11):1068.

10. Eliakim-Raz N, Robenshtok E, Shefet D, Gafter-Gvili A, Vidal L, Paul M, et al. Empiric antibiotic coverage of atypical pathogens for community-acquired pneumonia in hospitalized adults. Cochrane Database Syst Rev. 2012;(9): Cd004418.

11. Burki TK. Beta-lactam monotherapy is non-inferior to combination treatment for community-acquired pneumonia. Lancet Respir Med. 2015;3(5):347.

12. Prina E, Ranzani OT, Torres A. Community-acquired pneumonia. Lancet. 2015:386(9998):1097-108.

13. Crawford J, Dale DC, Lyman GH. Chemotherapy-induced neutropenia: risks, consequences, and new directions for its management. Cancer. 2004;100(2): 228-37.

14. Lopez-Pousa A, Rifa J, Casas de Tejerina A, Gonzalez-Larriba JL, Iglesias C, Gasquet JA, et al. Risk assessment model for first-cycle chemotherapy- induced neutropenia in patients with solid tumours. Eur J Cancer Care (Engl). 2010;19(5):648-55.

15. Lim WS, van der Eerden MM, Laing R, Boersma WG, Karalus N, Town Gl, et al. Defining community acquired pneumonia severity on presentation to hospital: an international derivation and validation study. Thorax. 2003;58(5): 377-82.

16. Klastersky J, Paesmans M, Rubenstein EB, Boyer M, Elting L, Feld R, et al. The multinational Association for Supportive Care in Cancer risk index: a multinational scoring system for identifying low-risk febrile neutropenic cancer patients. J Clin Oncol. 2000;18(16):3038-51.

17. Mandell LA, Wunderink RG, Anzueto A, Bartlett JG, Campbell GD, Dean NC, et al. Infectious Diseases Society of America/American Thoracic Society consensus guidelines on the management of community-acquired pneumonia in adults. Clin Infect Dis. 2007:44(Suppl 2):S27-72.

18. American Thoracic S. Infectious diseases Society of a. guidelines for the management of adults with hospital-acquired, ventilator-associated, and healthcare-associated pneumonia. Am J Respir Crit Care Med. 2005;171(4): 388-416.

19. Therasse P, Arbuck SG, Eisenhauer EA, Wanders J, Kaplan RS, Rubinstein L, et al. New guidelines to evaluate the response to treatment in solid tumors. European Organization for Research and Treatment of Cancer, National Cancer Institute of the United States, National Cancer Institute of Canada. J Natl Cancer Inst. 2000;92(3):205-16.

20. Miller AB, Hoogstraten $B$, Staquet $M$, Winkler A. Reporting results of cancer treatment. Cancer. 1981;47(1):207-14.

21. Eisenhauer EA, Therasse P, Bogaerts J, Schwartz LH, Sargent D, Ford R, et al. New response evaluation criteria in solid tumours: revised RECIST guideline (version 1.1). Eur J Cancer. 2009;45(2):228-47.

22. Daxboeck F, Krause R, Wenisch C. Laboratory diagnosis of mycoplasma pneumoniae infection. Clin Microbiol Infect. 2003:9(4):263-73.

23. van der Eerden MM, Vlaspolder F, de Graaff CS, Groot T, Jansen HM, Boersma WG. Value of intensive diagnostic microbiological investigation in low- and high-risk patients with community-acquired pneumonia. Eur J Clin Microbiol Infect Dis. 2005;24(4):241-9.

24. Lin E, Stanek RJ, Mufson MA. Lack of synergy of erythromycin combined with penicillin or cefotaxime against Streptococcus pneumoniae in vitro. Antimicrob Agents Chemother. 2003;47(3):1151-3.

25. Johansen HK, Jensen TG, Dessau RB, Lundgren B, Frimodt-Moller N. Antagonism between penicillin and erythromycin against Streptococcus pneumoniae in vitro and in vivo. J Antimicrob Chemother. 2000;46(6):973-80.

26. Dwyer R, Ortqvist A, Aufwerber E, Henriques Normark B, Marrie TJ, Mufson MA, et al. Addition of a macrolide to a ss-lactam in bacteremic pneumococcal pneumonia. Eur J Clin Microbiol Infect Dis. 2006;25(8):518-21.

27. Hughes WT, Armstrong D, Bodey GP, Bow EJ, Brown AE, Calandra T, et al. 2002 guidelines for the use of antimicrobial agents in neutropenic patients with cancer. Clin Infect Dis. 2002;34(6):730-51.

\section{Publisher's Note}

Springer Nature remains neutral with regard to jurisdictional claims in published maps and institutional affiliations.

\section{Ready to submit your research? Choose BMC and benefit from:}

- fast, convenient online submission

- thorough peer review by experienced researchers in your field

- rapid publication on acceptance

- support for research data, including large and complex data types

- gold Open Access which fosters wider collaboration and increased citations

- maximum visibility for your research: over $100 \mathrm{M}$ website views per year

At BMC, research is always in progress.

Learn more biomedcentral.com/submission 